involves very little difficulty. If the conjunctiva is inadvertently button-holed, it matters little. Special care must be taken that the strip of tarsus excised extends the whole width of the plate, and for this purpose it is often necessary to re-adjust the Desmarres' forceps. Four or five sutures are then inserted passing through the skin of the inferior lip of the wound and deep into upper lip of tarsus, and made taut. The ends of the sutures are left long and are fixed on the brow by sticking-plaster (Fig. 1). The upper lip of the skin flap.is undisturbed. Depending on the degree of entropion, a wide strip may be excised or the sutures placed nearer to the cilia. It is not necessary to bandage both eyes. The sutures are removed in four days.

Recently, most of these cases have been re-examined, and I have good reason to be pleased with results. In no instance has there been a recurrence of the entropion and the cosmetic result has been excellent.

It wotild seem, therefore, that here is an operation which presents the following advantages:-

1. It is easy to perform.

2. It gives a complete and permanent cure of entropion.

3. It gives an excellent cosmetic result. In fact the slight ptosis present in all these cases is rectified, giving a rather pleasing, alert appearance (Fig 2).

For these reasons, it is considered that this operation might be more widely known and practised.

\title{
A MODIFICATION OF ARRUGA'S SPECULUM
}

BY

\section{HUMPHREY NEAME}

LONDON

For operation on detachment of the retina, Arruga's speculum of plated metal was taken as a model and an instrument made of plastic material was designed $2 \mathrm{~mm}$. wider than the original, so as to give a larger field within the hollow of the retractor for the application of diathermy in detachment of the retina.

The figure shows the general appearance of the speculum. The plastic material is roughened for a small area on its convex surface and for a larger area in the hollow of the concave side. Light enters from a small battery or through a transformer from the main 


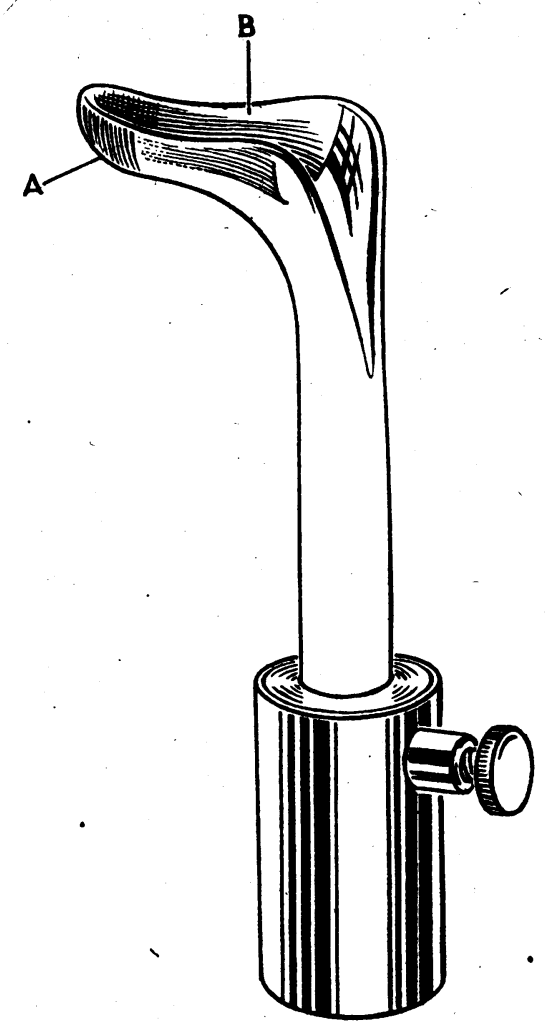

Neame's modification of Arruga's Speculum.

A. Outer surface
B. Inner surface roughened.

at the handle and is carried by internal reflection to the end of the instrument and is only dispersed when it meets the roughened surface. The retractor is, of course, quite free from heat, hence the proprietary name given to the instrument (Coldlite). The illumination of the area of the sclera requiring treatment is admirable and entirely obviates the presence of any shadows such as are inevitable to some extent in using a hand operating lamp. The greater width of the speculum is also an advantage and does not in the least make it difficult to use. The third and very definite advantage of this instrument is that it is a non-conductor of electricity.

This instrument is likely to supersede all other forms of retractor or speculum for this type of operation, for cases in which the area of the eye to be treated by diathermy is in the anterior part of the eye as well as in the posterior part. 